\title{
Piperacillin-tazobactam should be preferred to third- generation cephalosporins to treat wild-type inducible AmpC-producing Enterobacteriaceae in critically ill patients with hospital or ventilator- acquired pneumonia. A propensity score-matched analysis
}

Cédric Carrié ( $\nabla$ cedric.carrie@chu-bordeaux.fr)

Centre Hospitalier Universitaire de Bordeaux

Guillaume Bardonneau

Centre Hospitalier Universitaire de Bordeaux

Laurent Petit

Centre Hospitalier Universitaire de Bordeaux

Alexandre Ouattara

Centre Hospitalier Universitaire de Bordeaux

Didier Gruson

Centre Hospitalier Universitaire de Bordeaux

\section{Bruno Pereira}

Centre Hospitalier Universitaire de Bordeaux

Matthieu Biais

Centre Hospitalier Universitaire de Bordeaux

Research article

Keywords: Enterobacteriaceae; Piperacillin-tazobactam; Third-generation cephalosporins; Pulmonary infection; Intensive care

Posted Date: July 29th, 2019

DOI: https://doi.org/10.21203/rs.2.12028/v1

License: (a) (i) This work is licensed under a Creative Commons Attribution 4.0 International License. Read Full License 


\section{Abstract}

BACKGROUND The aim of this study was to compare the rate of therapeutic failure and emergence of resistance in critically ill patients treated by third-generation cephalosporins (3GCs) or piperacillintazobactam (PTZ) for wild-type AmpC-producing Enterobacteriaceae pulmonary infections. METHODS In a multicenter retrospective cohort study over a 4-year period, all patients treated for a pulmonary infection related to wild-type AmpC-producing Enterobacteriaceae who received documented antibiotic therapy with 3GCs or PTZ after less than 48 hours of empirical antibiotic therapy were eligible. The main outcome was the rate of therapeutic failure, defined by an impaired clinical response under treatment and/or a relapse of pulmonary infection related to the same pathogen. The secondary outcome was a secondary acquisition of derepressed cephalosporinase-producing Enterobacteriaceae. RESULTS Over the study period, 244 patients were included; 56 (23\%) experienced therapeutic failure and 19 (8\%) experienced secondary acquisition of resistance. In the non-adjusted cohort, the rate of therapeutic failure and emergence of resistance were significantly higher in the $3 \mathrm{GCs}$ group (32 vs. $18 \%, p=0.011$ and $13 \mathrm{vs.} 5 \%$, $p=0.035$, respectively). In the propensity score-matched population, the 3GCs group was associated with higher rates of therapeutic failure $(H R=1.61[1.27-2.07])$. The secondary de-escalation to $3 G C s$ after $48 \mathrm{~h}$ of PTZ as a first-line antibiotic therapy was not associated with increased rate of emergence of resistance. CONCLUSION Our study confirms that third-generation cephalosporins should be avoided as first-line antibiotic therapy in wild-type AmpC-producing Enterobacteriaceae pulmonary infections.

\section{Background}

In the intensive care settings, Enterobacteriaceae are among the most common pathogens involved in hospital-acquired pneumonia or ventilator-associated pneumonia (HAP - VAP) [1]. A peculiar phenotype is observed in Enterobacter spp., Serratia marcescens, Citrobacter freundii, Hafnia alvei or Morganella morganii, characterized by inducible resistance mediated by a chromosomal Amp C $\beta$-Lactamase [2]. In this context, appropriate antibacterial exposure is essential to avoid the emergence of highly-resistant derepressed mutants, responsible for higher mortality and healthcare-related costs [3].

However, controversies remain regarding the choice of antimicrobial agents that can be used to treat wildtype inducible AmpC-producing Enterobacteriaceae in critically ill patients with HAP-VAP. According to former studies, third-generation cephalosporins (3GCs) should be avoided owing to a higher risk for selecting resistant mutants $[4,5]$. Piperacillin-tazobactam (PTZ), a weak inducer of AmpC $\beta$-Lactamases, may be an attractive alternative in this context [6].

On the other hand, the level of evidence supporting such a strategy is poor. Indeed, most of those studies had limited power to explore the relationship between the type of antibiotic therapy, the highly variable incidence of resistant mutant selection and the low rate of clinical failure [4-7]. Moreover, other studies found a similar rate of emergence of antibiotic resistance among treatment groups, suggesting an identical selective pressure between 3GCs and PTZ [8]. In this context, some authors argued that although selection of resistant mutants during treatment is a serious event, the risk may be over- 
estimated and offset by the overall cure rates and the ecological impact of using 3GCs in the light of therapeutic alternatives [9].

The main objective of this study was to compare the rate of therapeutic failure and emergence of resistance in critically ill patients treated by 3GCs or PTZ for a first episode of wild-type AmpC-producing Enterobacteriaceae pneumonia.

\section{Methods}

\section{Study design and population}

This retrospective cohort study was conducted in four Intensive Care Units (ICUs) in two University Hospitals (CHU Pellegrin, Bordeaux, France and CHU Haut-Lévêque, Pessac, France) over a 4-year period (from January 2015 to December 2018). Ethical approval was obtained from the Institutional Review Board (GP-CE2019-08) which waived the need for written consent. The patients and/or next of kin were informed about the inclusion of their anonymized data in the database, and none declined participation.

Patients were eligible for inclusion if they met all the following criteria: i) age $\geq 18$, ii) treated for a HAPVAP defined by usual criteria [10], (ii) with quantitative culture results positive for wild-type AmpCproducing Enterobacteriaceae (Enterobacter spp., Serratia marcescens, Citrobacter freundii, Hafnia alvei or Morganella morganii) [2], (iv) who received documented antibiotic therapy with 3GCs (Ceftriaxone, Cefotaxime or Ceftazidime) or PTZ. Patients who received less than 48 hours of empirical antibiotic therapy with secondary de-escalation to 3GCs were considered in the 3GCs group. Patients who received more than 48 hours of PTZ as an empirical antibiotic therapy with secondary de-escalation to 3GCs were considered in the PTZ group. Patients who received more than 48 hours of empirical antibiotic therapy with another $\beta$-Lactam (cefepime, meropenem) were excluded. Other exclusion criteria were absence of HAP-VAP criteria, inappropriate antibiotic therapy (natural or acquired resistances to 3GCs or PTZ) or decision for limitation or withdrawal of life-sustaining therapies.

\section{Antibiotic protocol}

During all the study period, diagnosis of HAP-VAP was defined as new or progressive pulmonary infiltrates in plain chest radiograph, persistent fever or hypothermia, leukopenia or leukocytosis and at least two of the following criteria: cough or dyspnea, purulent bronchial secretions, oxygen requirement) [10]. Diagnosis of HAP-VAP was secondarily documented by quantitative cultures ( $\geq 10^{4} \mathrm{UFC} / \mathrm{mL}$ for BALs, $\geq 10^{6} \mathrm{UFC} / \mathrm{mL}$ for endotracheal aspirations and $\geq 10^{7}$ for non-invasive sputum samples).

Empirical antibiotic therapyfor clinically suspected HAP-VAP was determined by the attending physician, with guidance from a local protocol in accordance with international recommendations [11]. In our local practice and according to published data in critically ill patients with normal renal function, standard dosing regimens of $\beta$-lactam therapy were Piperacillin-Tazobactam $(16 \mathrm{~g} /$ day, either continuously or by extended infusion), Ceftazidime or Cefepime ( $6 \mathrm{~g} /$ day continuously or by extended infusion), Cefotaxime 
( $2 \mathrm{~g} \mathrm{IV} \mathrm{q8hr)} \mathrm{or} \mathrm{Ceftriaxone} \mathrm{(2g} \mathrm{once/day).} \mathrm{Dose} \mathrm{adjustment} \mathrm{was} \mathrm{allowed} \mathrm{in} \mathrm{patients} \mathrm{with} \mathrm{renal} \mathrm{failure} \mathrm{or}$ augmented renal clearance [12]. A loading dose was systematically given before beginning continuous infusion. Aminoglycosides (gentamycin, amikacin) were administered in patients with septic shock or ARDS at time of HAP-VAP and subsequently dosed by therapeutic drug monitoring. De-escalation of empiric antibiotic therapy was assessed whenever possible after identification of the causative microorganism and reception of the antibiotic susceptibilities.

\section{Study end-points}

The main outcome investigated in this study was the rate of therapeutic failure within 28 days and/or end-of-hospitalization defined by i) a death directly attributable to the HAP-VAP, ii) an impaired clinical response (worsening, persistent or recurrent clinical, biological and radiological features of the initial infection) with a need for escalating antibiotics despite an adequate antibiotic therapy and/or iii) a new pulmonary infection needing the resumption of antibiotic therapy with the same pathogen growing at a significant concentration from a second sample (HAP - VAP relapse) $[12,13]$. Superinfections due to new causative pathogens were not considered as therapeutic failure. The primary outcome was assessed independently by at least two of the investigators (GB, CC) and discrepancies were resolved by discussion between the authors. The secondary outcome was the emergence of resistant mutant, defined by a second sample positive for derepressed cephalosporinase-producing Enterobacteriaceae. Other secondary endpoints were duration of mechanical ventilation, ICU or hospital length of stay, in-hospital mortality and incidence of documented adverse drug events.

\section{Statistical analysis}

Results are expressed as mean \pm standard deviation or median ( $25 \%$ to $75 \%$ interquartile range) for continuous variables and as absolute or relative frequencies for categorical variables. The data distribution was analyzed by a Kolmogorov-Smirnov test. Unadjusted odd ratios (OR) with $95 \%$ confidence interval $(95 \% \mathrm{Cl})$ were determined using univariate logistic regression. Comparisons between continuous variables were performed using the Student $t$ test or the Mann-Whitney test and categorical variables were compared using the chi-square test or Fisher's exact test as appropriate. Multivariate analysis was then performed to determine relevant covariates associated with therapeutic failure and emergence of resistance.

To adjust for potential baseline differences between the treatment groups, a propensity score analysis was performed to predict the conditional probability for an individual patient to receive 3GCs or PTZ [14]. The covariates included in the propensity score model were known to be clinically relevant and fixed according to univariate analysis. A particular attention was paid to interactions and multicollinearity. Propensity score matching was performed with a calliper width of 0.2 . As previously described, bootstrapping simulations (5000 simulations) were used to develop propensity score-matched pairs without replacement $(1: 1$ match) $[15,16]$. Cox proportional-hazards regression analysis was performed to compare the clinical outcome between patients treated by 3GCS or PTZ. 
Statistical analyses were performed using XLSTAT 2015 for Windows (Addinsoft Paris, France) and Stata software (version 13; StataCorp, USA).

\section{Results}

\section{Characteristics of the population}

During the study period, 591 patients were included in the present study. Among these patients, 347 were excluded (no criteria for HAP-VAP, $N=160$; cefepime or meropenem for more than 48 hours, $N=100$; limitation or withdrawal of life-sustaining therapies, $N=87$ ). Finally, 244 patients were included for analysis: 157 patients in the PTZ group and 87 patients in the 3GCs group (Ceftriaxone, $N=45$; Cefotaxime, $\mathrm{N}=34$ or Ceftazidime, $\mathrm{N}=8$ ). The main characteristics of the population are shown in Table 1 .

Among these patients, 56 (23\%) experienced therapeutic failure (impaired clinical response during treatment, $\mathrm{N}=34 ;$ HAP-VAP relapse, $\mathrm{N}=22$ ) and 19 (8\%) experienced secondary acquisition of resistance. Patients experiencing therapeutic failure were likely to have a longer MV duration (23 [12-30] vs. 8 [218 ] days, $p<0.0001)$, longer ICU length of stay (31 [23-43] vs. 16 [10-28] days, $p<0.0001)$ and higher mortality rate (23 vs. $7 \%, p=0.001)$.. The rates of therapeutic failure and emergence of resistance according to the type of pathogen are reported in Supplementary Data.

\section{Association between therapeutic failure and treatment group}

In the non-adjusted cohort of patients, the rate of therapeutic failure was significantly higher in the 3GCs group (32 vs. $18 \%, p=0.011$ ).. Univariate time-to-event analysis is depicted figure 1. Multivariate analysis demonstrated a significant association between $3 \mathrm{GCs}$ and therapeutic failure (Table $2 A$ ).. Of note, the association remained significant in patients who received $3 \mathrm{GCs}$ as a first-line antibiotic therapy (OR = $2.28[1.22-4.26], p=0.009$ ) but not in patients who received less than 48 hours of empirical antibiotic therapy with secondary de-escalation to $3 \mathrm{GCs}(\mathrm{OR}=1.13$ [0.41-3.14], $p=0.82)$.. The use of PTZ as a first-line antibiotic therapy was associated with a lower rate of therapeutic failure ( $O R=0.38$ [0.21-0.70], $p=0.002)$.. The secondary de-escalation to $3 G C$ s after $48 \mathrm{~h}$ of PTZ as a first-line antibiotic therapy was not associated with increased rate of therapeutic failure. Clinical data at admission, characteristics of HAP-VAP, type of pathogen, inoculum size or combined antimicrobial therapy were not associated with therapeutic failure.

In the propensity score-matched population, the 3GCs group was associated with higher rates of therapeutic failure (Figure 2).. The covariates included in the propensity score model were: age, reason for admission, early HAP-VAP, modified SOFA score, previous antibiotic exposure, augmented renal clearance, combined antimicrobial therapy and Enterobacter spp. The data were adjusted for propensity score, $\mathrm{PaO}_{2} / \mathrm{FiO}_{2}$ ratio and use of vasopressors.

3. Association between emergence of resistance and treatment group 
In the non-adjusted cohort of patients, the rate of emergence of derepressed cephalosporinase was significantly higher in the $3 \mathrm{GCs}$ group ( $13 \mathrm{vs.} 5 \%, p=0.035)$.. Multivariate analysis showed that the $3 \mathrm{GCs}$ group was associated with secondary emergence of derepressed cephalosporinase (Table 2B).. Of note, the association remained significant in patients who received $3 \mathrm{GCs}$ as a first-line antibiotic therapy (OR = 2.59 [1.03-6.55], $p=0.043$ ) but not in patients who received less than 48 hours of empirical antibiotic therapy with secondary de-escalation to $3 \mathrm{GCs}(\mathrm{OR}=1.35$ [0.33-5.53], $p=0.70)$.. The use of PTZ as a first-line antibiotic therapy was associated with fewer emergence of resistance $(O R=0.32$ [0.13-0.82], $p$ $=0.02)$.. The secondary de-escalation to $3 G C s$ after $48 \mathrm{~h}$ of PTZ as a first-line antibiotic therapy was not associated with increased rate of emergence of resistance. Clinical data at admission, characteristics of HAP-VAP, type of pathogen, inoculum size or combined antimicrobial therapy were not associated with emergence of resistance.The mean MIC for ceftriaxone was significantly higher in patients experiencing secondary emergence of resistance $(0.6 \pm 0.2$ vs. $0.5 \pm 0.3, p=0.013)$..

In the propensity score-matched population, there was a non-significant trend toward higher rates of emergence of resistance in the $3 \mathrm{GCs}$ group (OR $=2.85$ [0.90 - 8.97], $p=0.07)$.. The data were adjusted for propensity score, $\mathrm{PaO}_{2} / \mathrm{FiO}_{2}$ ratio and MIC for ceftriaxone.

\section{Discussion}

Our results suggested that patients treated by $3 \mathrm{GCs}$ as first-line antibiotic therapy or after less than 48 hours of empirical antibiotic therapy for a first episode of wild-type AmpC-producing Enterobacteriaceae HAP-VAP had higher risk of therapeutic failure compared to patients treated by PTZ. This is of major concern as the incidence of therapeutic failure was high in this population, associated with longer MV durations, longer ICU length of stay and higher mortality [3].

In patients treated for wild-type AmpC-producing Enterobacteriaceae infections, the selection of resistant mutant should be one of the first hypotheses to rule out when infection does not improve or relapse after a first-line $\beta$-Lactam therapy [2]. In this context, Chow et al. first reported an association between prior 3GCs exposure and secondary emergence of resistance in Enterobacter bloodstream infections, but the sample size was small (6 out of 31 patients) [4]. Similar outcomes were reported in larger cohorts of patients, also the rate of resistant mutant selection were significantly lower [5, 7]. However, those studies were impaired by the wide range of patients and infections, the various dosing regimens for 3GCS or PTZ or the lack of assessment of clinical outcome. Although controversies remain [8], 3GCs are usually not recommended as first line therapy, even when susceptible in vitro [17].

On the other hand, the association between resistant mutant selection and clinical failure is not straightforward [18]. In the present study, the therapeutic failure rate was significantly higher than the secondary emergence of derepressed cephalosporinase, suggesting that emergence of resistant organisms is neither the only nor the more common cause of therapy failure. Moreover, the main difference between treatment groups occurred early, responsible for impaired clinical response during treatment, while the rate of HAP-VAP relapse was not statistically different. In this context, antibiotic 
pharmacokinetic and pharmacodynamic (PK/PD) issues could explain these discrepancies, especially in patients with severe HAP-VAP, high distribution volume and/or augmented renal clearance [19]. Other factors, such as the bacterial inoculum size and the rate of penetration of antimicrobial agents should also be considered [20]. On the other hand, the association between 3GCs and therapeutic failure remained after propensity score-matched analysis including confounding covariates such as SOFA score, combined antimicrobial therapy and augmented renal clearance. This issue thus warrants further investigations.

Moreover, clinical outcome of patients may be different when 3GCs are given as a first-line antibiotic therapy or after antibiotic de-escalation. Although our study is underpowered for a proper subgroup analysis, secondary de-escalation to 3GCs after a first-line empirical antibiotic therapy was not associated with increased rate of therapeutic failure. The level of evidence supporting such a strategy is however conflicting. In a multicenter non-blinded randomized non-inferiority trial, a strategy based on deescalation of antibiotics resulted in prolonged duration of ICU stay and increased number of superinfections as compared to the continuation of the empirical antimicrobial treatment [21]. Although microbiological data were not reported, the rate of relapse (superinfection episodes related to the same bacteria) was significantly lower in the continuation group ( $27 \mathrm{vs.} 11 \%, p=0.03$ ). The continuation of broader spectrum antibiotic therapy for a shorter duration, ensuring optimization of antibiotic levels and biomarker monitoring, may be an alternative promising strategy to decrease the selection pressure [22].

Several limitations should be reported. The main limitation relied on the retrospective design that could lead to selection and interpretation bias. However, the propensity score matching allowed reducing the effects of confounding covariates between treatment groups. Moreover, antibiotic therapy was not protocolized and treatment groups were arbitrarily allocated according to the type and duration of empirical antibiotic exposure. Finally, there is no consensus statement on the definition of treatment failure. Sustained defervescence, clinical stability and reduction of SOFA score in a 48-72-h timeframe are the variables that are considered in several studies and seem to better reflect clinical response [19]. However, external validation of the study end-point was not blinded to the allocated treatment group and therapeutic drug monitoring is lacking to rule out under-dosing of antimicrobial agents.

\section{Conclusions}

In conclusion, our study suggests that third-generation cephalosporins should be avoided as first-line antibiotic therapy wild-type AmpC-producing Enterobacteriaceae HAP-VAP. Further data are needed for determination of risk factors for developing infection related to wild-type AmpC-producing Enterobacteriaceae in order to better guide empirical antibiotic therapy in patients with early VAP-HAP. Whether a secondary de-escalation may be safe and effective to prevent HAP-VAP relapse remains to be documented in this population.

\section{Abbreviations}


3GCs $=$ third-generation cephalosporins

HAP = hospital-acquired pneumonia

$\mathrm{HR}=$ Hazard ratio

$\mathrm{ICU}=$ Intensive Care Unit

$\mathrm{MV}=$ Mechanical ventilation

PTZ = piperacillin-tazobactam

$\mathrm{OR}=$ Odds ratio

SOFA $=$ Sepsis-related Organ Failure

VAP $=$ ventilator-associated pneumonia

\section{Declarations}

Ethics approval and consent to participate

Ethical approval was obtained from the Institutional Review Board (Comité publication, Centre Hospitalier Universitaire de Bordeaux, number GP-CE2019-08) which waived the need for written consent. The patients and/or next of kin were informed about the inclusion of their anonymized data in the database, and none declined participation.

Consent for publication

Not applicable

Availability of data and material

The datasets used and/or analysed during the current study are available from the corresponding author on reasonable request.

Competing interests

The authors declare that they have no competing interests.

Funding

Only departmental funds were used for this study. No external funds were obtained.

Authors' contributions 
CC designed the study and wrote the manuscript. GB was responsible for the integrity of the data collection and the accuracy of the data analysis. CC and GB had full access to all the data in the study and assumed full responsibility for the integrity of the submission as a whole. MB and BP helped to provide statistical advice on the design of the study, analyze the data and write the manuscript. AO, LP and DG personally supervised the extraction of the data, confirmed an understanding of the statistical methods used for the efficacy analysis, and confirmed that the objectives and procedures of the study were honestly disclosed. All authors read and approved the final manuscript.

Acknowledgements

Not applicable

Authors' information (optional)

Not applicable

\section{References}

1. Luyt CE, Hékimian G, Koulenti D, Chastre J. Microbial cause of ICU-acquired pneumonia: hospitalacquired pneumonia versus ventilator-associated pneumonia. Curr Opin Crit Care. 2018; 24(5):3328.

2. Ruppé É, Woerther PL, Barbier F. Mechanisms of antimicrobial resistance in Gram-negative bacilli. Ann Intensive Care. 2015; 5(1):61.

3. Cosgrove SE, Kaye KS, Eliopoulous GM, Carmeli Y. Health and economic outcomes of the emergence of third-generation cephalosporin resistance in Enterobacter species. Arch Intern Med. 2002; 162(2):185-90.

4. Chow JW, Fine MJ, Shlaes DM, Quinn JP, Hooper DC, Johnson MP, Ramphal R, Wagener MM, Miyashiro DK, Yu VL. Enterobacter bacteremia: clinical features and emergence of antibiotic resistance during therapy. Ann Intern Med. 1991; 115(8):585-90.

5. Kaye KS, Cosgrove S, Harris A, Eliopoulos GM, Carmeli Y. Risk factors for emergence of resistance to broad-spectrum cephalosporins among Enterobacter spp. Antimicrob Agents Chemother. 2001; 45(9):2628-30.

6. Cheng L, Nelson BC, Mehta M, Seval N, Park S, Giddins MJ, Shi Q, Whittier S, Gomez-Simmonds A, Uhlemann AC. Piperacillin-Tazobactam versus Other Antibacterial Agents for Treatment of Bloodstream Infections Due to AmpC $\beta$-Lactamase-Producing Enterobacteriaceae. Antimicrob Agents Chemother. 2017; 61(6).

7. Choi SH, Lee JE, Park SJ, Choi SH, Lee SO, Jeong JY, Kim MN, Woo JH, Kim YS. Emergence of antibiotic resistance during therapy for infections caused by Enterobacteriaceae producing AmpC beta-lactamase: implications for antibiotic use. Antimicrob Agents Chemother. 2008; 52(3):9951000. 
8. Schwaber MJ, Graham CS, Sands BE, Gold HS, Carmeli Y. Treatment with a broad-spectrum cephalosporin versus piperacillin-tazobactam and the risk for isolation of broad-spectrum cephalosporin-resistant Enterobacter species. Antimicrob Agents Chemother. 2003; 47(6):1882-6.

9. Goldstein FW. Cephalosporinase induction and cephalosporin resistance: a longstanding misinterpretation. Clin Microbiol Infect. 2002; 8(12):823-5.

10. Leone M, Bouadma L, Bouhemad B, Brissaud O, Dauger S, Gibot S, Hraiech S, Jung B, Kipnis E, Launey Y, Luyt CE, Margetis D, Michel F, Mokart D, Montravers P, Monsel A, Nseir S, Pugin J, Roquilly A, Velly L, Zahar JR, Bruyère R, Chanques G. Hospital-acquired pneumonia in ICU. Anaesth Crit Care Pain Med. 2018; 37(1):83-98.

11. Carrie C, Bentejac M, Cottenceau V, Masson F, Petit L, Cochard JF, Sztark F. Association between augmented renal clearance and clinical failure of antibiotic treatment in brain-injured patients with ventilator-acquired pneumonia: A preliminary study. Anaesth Crit Care Pain Med. 2018;37(1):35-41

12. Besnard T, Carrié $C$, Petit L, Biais M. Increased dosing regimens of piperacillin-tazobactam are needed to avoid subtherapeutic exposure in critically ill patients with augmented renal clearance. Crit Care. 2019; 23(1):13.

13. Carrié C, Petit L, d'Houdain N, Sauvage N, Cottenceau V, Lafitte M, Foumenteze C, Hisz Q, Menu D, Legeron R, Breilh D, Sztark F. Association between augmented renal clearance, antibiotic exposure and clinical outcome in critically ill septic patients receiving high doses of $\beta$-lactams administered by continuous infusion: a prospective observational study. Int J Antimicrob Agents. 2018;51(3):443-9.

14. Austin PC. An Introduction to Propensity Score Methods for Reducing the Effects of Confounding in Observational Studies. Multivar Behav Res. 2011;46: 399-424

15. Austin PC. The relative ability of different propensity score methods to balance measured covariates between treated and untreated subjects in observational studies. Med Decis Mak Int J Soc Med Decis Mak. 2009;29: 661-77

16. Austin PC, Small DS. The use of bootstrapping when using propensity-score matching without replacement: a simulation study. Stat Med. 2014;33: 4306-19

17. Livermore DM, Brown DF, Quinn JP, Carmeli Y, Paterson DL, Yu VL. Should third-generation cephalosporins be avoided against AmpC-inducible Enterobacteriaceae? Clin Microbiol Infect. 2004; 10(1):84-5.

18. Milatovic D, Braveny I. Development of resistance during antibiotic therapy. Eur J Clin Microbiol. 1987; 6(3):234-44.

19. Bassetti M, Montero JG, Paiva JA. When antibiotic treatment fails. Intensive Care Med. 2018; 44(1):73-5.

20. Boselli E, Breilh D, Rimmelé T, Guillaume C, Xuereb F, Saux MC, Bouvet L, Chassard D, Allaouchiche B. Alveolar concentrations of piperacillin/tazobactam administered in continuous infusion to patients with ventilator-associated pneumonia. Crit Care Med. 2008; 36(5):1500-6.

21. Leone M, Bechis C, Baumstarck K, Lefrant JY, Albanèse J, Jaber S, Lepape A, Constantin JM, Papazian L, Bruder N, Allaouchiche B, Bézulier K, Antonini F, Textoris J, Martin C. De-escalation 
versus continuation of empirical antimicrobial treatment in severe sepsis: a multicenter non-blinded randomized non-inferiority trial. Intensive Care Med. 2014; 40(10):1399-408.

22. Dimopoulos G, Matthaiou DK. Duration of therapy of ventilator-associated pneumonia. Curr Opin Infect Dis. 2016; 29(2):218-22.

\section{Tables}

Due to technical limitations, the Table(s) are only available as a download in the supplemental files section.

\section{Figures}

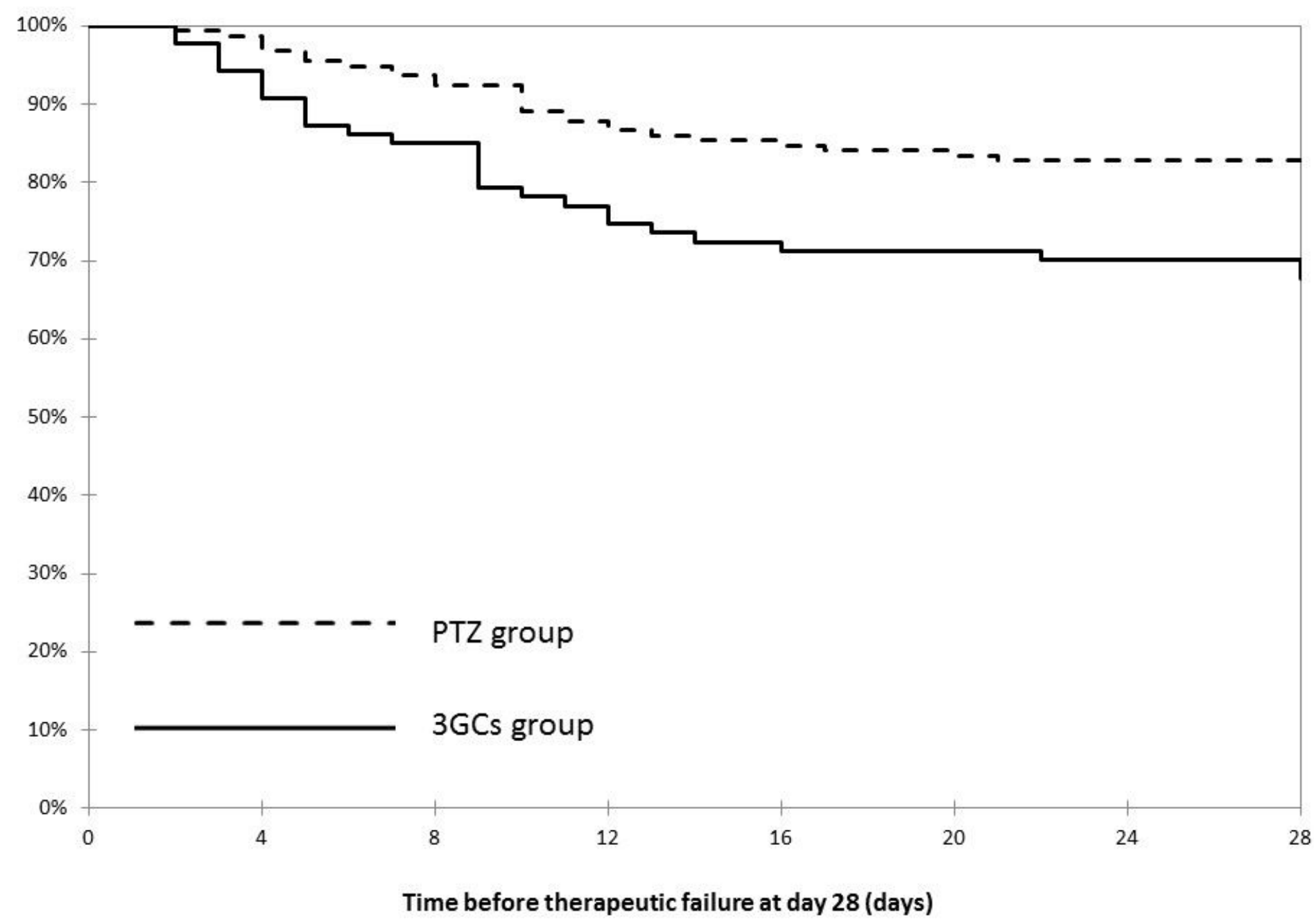

\section{Figure 1}

Kaplan-Meier survival curves of patients treated by 3GCs (full line) or PTZ (dotted line) for a first episode of wild-type AmpC-producing Enterobacteriaceae pneumonia. 


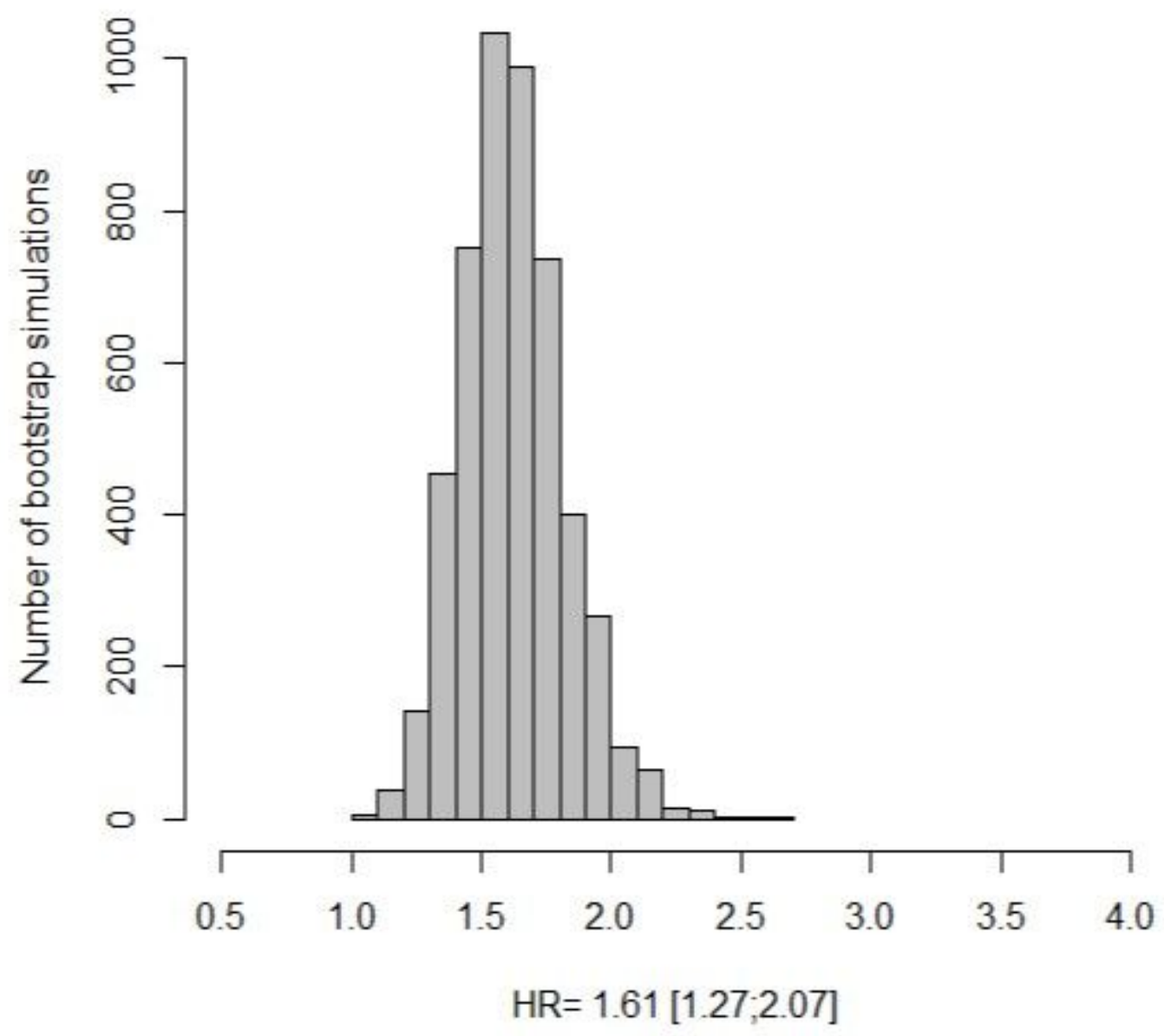

Figure 2

Distribution of Hazard-ratios adjusted on propensity score, $\mathrm{PaO} 2 / \mathrm{FiO} 2$ ratio and use of vasopressors after bootstrapping simulations (5000 simulations).

\section{Supplementary Files}

This is a list of supplementary files associated with this preprint. Click to download.

- SuppIData1.pdf

- SuppIData2.pdf

- Table1.jpg

- Table2.jpg 